\title{
Frictional drag effect between massless and massive fermions in single-layer/bilayer graphene heterostructures
}

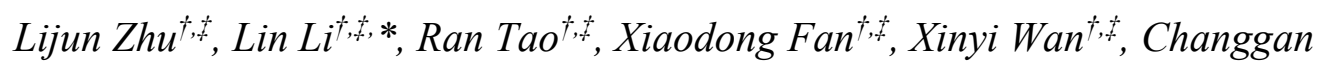
$\operatorname{Zeng}^{\dagger, t, *}$

$\dagger$ International Center for Quantum Design of Functional Materials (ICQD), Hefei

National Laboratory for Physical Sciences at the Microscale, and Synergetic

Innovation Center of Quantum Information and Quantum Physics, University of Science and Technology of China, Hefei, Anhui 230026, China

${ }^{*}$ CAS Key Laboratory of Strongly-Coupled Quantum Matter Physics, and Department of Physics, University of Science and Technology of China, Hefei, Anhui 230026, China

* Corresponding authors: cgzeng@ustc.edu.cn, lilin@ustc.edu.cn 


\section{Supplementary notes:}

\section{Note 1. Experimental sections}

Preparation of the vertical heterostructure: SLG and BLG flakes were mechanically exfoliated from Kish graphite and transferred onto $\mathrm{Si} / \mathrm{SiO}_{2}$ substrates. The number of layers and the quality of flakes were identified by a combination of optical microscopy ${ }^{1}$ and Raman spectroscopy, ${ }^{2,3}$ and the results are depicted in Figures S1a,b. The vertical heterostructure consisting of five layers of exfoliated layered materials (BN-SLG-BNBLG-BN), were then assembled onto a $\mathrm{SiO}_{2} / \mathrm{Si}$ substrate via the typical van der Waals assembly technique ${ }^{4}$ using a homemade manipulation stage (see Figure S1c), followed by annealing at $350{ }^{\circ} \mathrm{C}$ in $\mathrm{Ar} / \mathrm{H}_{2}$ atmosphere to remove chemical residues.

Electrode fabrication: SLG/BLG heterostructure was shaped into multi-terminal crossed electrode geometry by electron-beam lithography and reactive ion etching, as marked by the dashed lines in Figure $1 \mathrm{~b} . \mathrm{Cr} / \mathrm{Pd} / \mathrm{Au}$ films with thicknesses of $1 \mathrm{~nm} / 7$ $\mathrm{nm} / 45 \mathrm{~nm}$ were deposited on both of the graphene layers serving as 1D contact electrodes by electron beam evaporation. The device was then annealed at $350{ }^{\circ} \mathrm{C}$ in $\mathrm{Ar} / \mathrm{H}_{2}$ atmosphere in order to remove chemical residues and to get better contact between graphene and electrodes.

Transport measurements: The interlayer drag measurements, and the layer transport properties were performed in an Oxford Instruments ${ }^{4} \mathrm{He}$ cryostat. Keithley 6220/6221 and 2182A were employed to supply the currents and measure the voltages, respectively. The DC current for the drag measurements was set to be $1 \mu \mathrm{A}$ based on the linear $V_{\text {drag }}$ - Idrive curves (as typically shown in Figure S2b). To eliminate the voltage background of $2182 \mathrm{~A}$, the current was applied using a bipolar mode, and the voltage was obtained by taking an average of measured voltages for positive and negative currents.

\section{Note 2. Validity of our drag measurements}

To ensure the validity of our drag measurements, the Onsager reciprocity relations 
were checked via interchanging the drive and drag layers, as schematically shown in Figure S2a. Figure S2b demonstrates the obtained $V_{\text {drag }}-I_{\text {drive }}$ curves measured at 140 $K$. For these two opposite setups, $V_{\text {drag }}$ show well-defined linear response to $I_{\text {drive }}$ in the measured range. The values of $R_{\text {drag }}$ extracted from the slope of a linear fit, are nearly the same. Furthermore, the evolution of $R_{\text {drag }}$ as a function of $V_{\mathrm{BG}}$ was also measured, and nearly identical results were obtained for these two setups, further demonstrating the Onsager reciprocity relations are satisfied.

We also checked the $V_{\text {drag }}-I_{\text {drive }}$ relationship at different temperatures by using setup 1. Figure S3 shows the results obtained at several typical temperatures. It is found that the good liner $V_{\text {drag }}-I_{\text {drive }}$ relation is obeyed at temperatures above $70 \mathrm{~K}$, below which the fluctuations of $V_{\text {drag }}$ hinder the extraction of accurate $R_{\text {drag }}$ via linear fitting. Such deviation is a manifestation of mesoscopic drag fluctuations, which has also been observed in drag measurement of graphene-GaAs heterostructures. ${ }^{9}$ The drag fluctuations emerge at low temperatures as a consequence of phase coherent quantum transport, which share the same origin as the universal conductance fluctuations in the conventional layer conductance. . $^{5-8,10,11}$

As a typical quantum interference effect, such drag fluctuations should possess reproducibility, which has indeed been observed in previous study. ${ }^{6}$ We thus conducted gate-voltage dependent $R_{\text {drag }}$ measurements at low temperatures by using a constant $I_{\text {drive }}$ of $1 \mu \mathrm{A}$, and the typical results at $1.5 \mathrm{~K}$ are shown in Figure $\mathrm{S} 4$. The results clearly show that the fluctuations dominate the drag signal, and result in an alternating sign of $R_{\text {drag. }}$ Furthermore, nearly identical characteristics of the drag fluctuations were observed in two different scans, further validating the fluctuation picture.

\section{Note 3. Validity of carrier density tuning via interlayer-gate}

In our study, the carrier densities of the SLG and BLG layers are tuned by combing back-gate voltage $\left(V_{\mathrm{BG}}\right)$ and interlayer-gate voltage $\left(V_{\text {int }}\right)$, with $\mathrm{SiO}_{2}$ and $\mathrm{BN}$ serve as dielectric layers, respectively. Similar gate configuration was used previously for drag measurements and Fermi energy probing in graphene-based double layer heterostructures. $^{8,12}$ 
Note that the use of interlayer bias can induce a transverse electric field, which will lead to a band gap opening and the corresponding flat E-k dispersion near the charge neutrality point (CNP). ${ }^{13-15}$ Nevertheless, in this regime, our observations are similar to previous positive drag studies in which standard dual-gated configurations were used, ${ }^{10,16}$ implying the negligible effect of gap opening near the CNP. More importantly, we focused on the regime away from the CNP (carrier density $n>6 \times 10^{11} \mathrm{~cm}^{-2}$ ), in which novel carrier density dependent characteristics of drag resistance are discovered. Even if the flatted band regime is involved, the BLG layer remains a massive system. Thus the main conclusion here, i.e., the novel carrier density dependent drag originates from the interaction between massless and massive fermions, should still hold. Moreover, compared with the dual-gated configuration with top-gate, the device fabrication for interlayer-gate in the present study is relatively simple. 


\section{Supplementary Figures:}
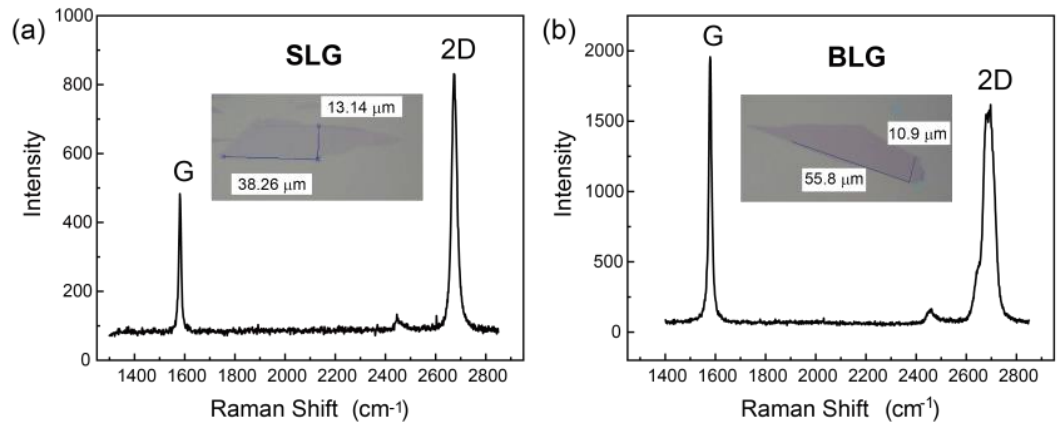

(c)
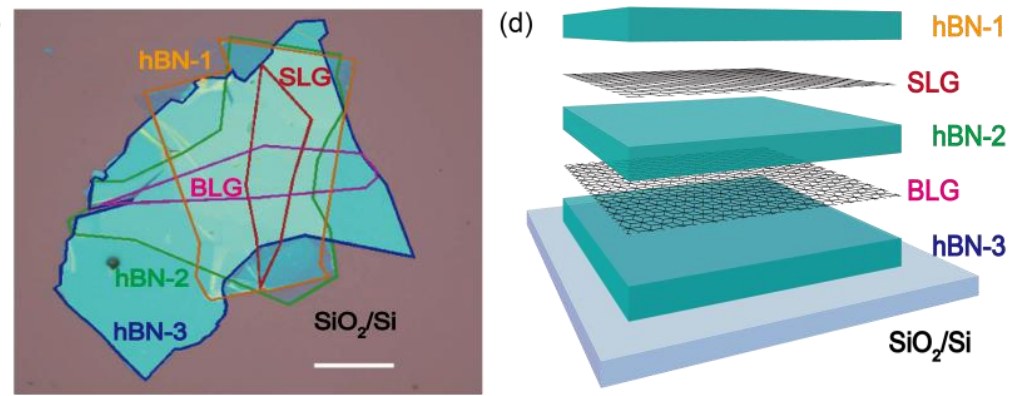

Figure S1. Preparation and characterization of SLG/BLG heterostructure. Raman spectra of (a) SLG and (b) BLG after exfoliated onto $\mathrm{SiO}_{2} / \mathrm{Si}$ substrates. (c) Optical image and (d) schematic diagram of SLG/BLG heterostructure assembled on a $\mathrm{SiO}_{2} / \mathrm{Si}$ substrate, the scale bar in (c) is $20 \mu \mathrm{m}$.

(a)

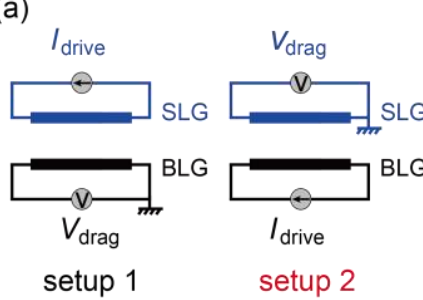

(b)

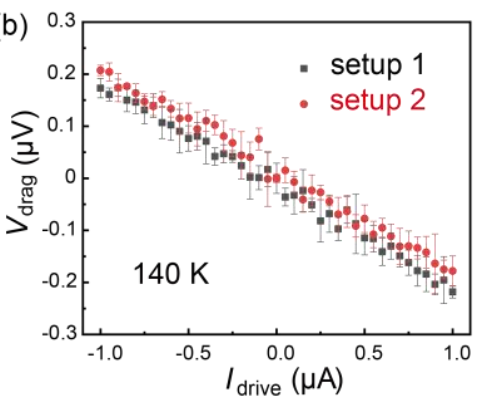

(c)

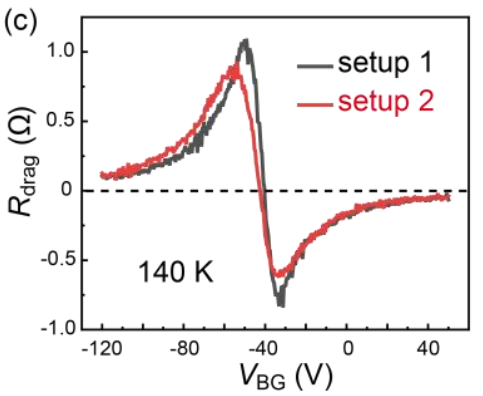

Figure S2. Verification of Onsager reciprocity relations. (a) Schematic diagrams for two different setups of drag measurements. (b) Comparison of $V_{\text {drag }}$ vs $I_{\text {drive }}$ curves for two different setups measured at $140 \mathrm{~K} ; V_{\mathrm{BG}}$ and $V_{\text {int }}$ were set to be 0 during the measurements. (c) $R_{\text {drag }}$ vs $V_{\mathrm{BG}}$ for two different setups measured at $140 \mathrm{~K}, V_{\text {int }}=0 \mathrm{~V}$ during the measurements. 

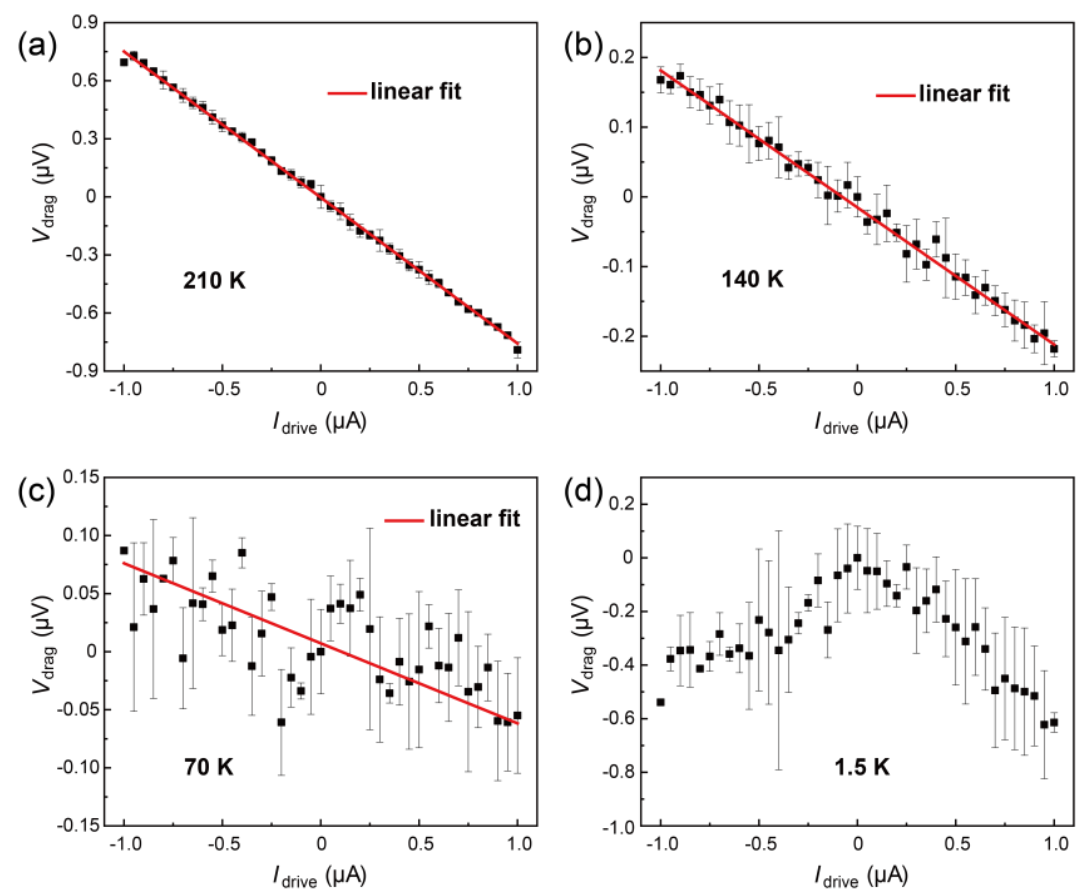

Figure S3. $V_{\text {drag }}-I_{\text {drive }}$ characteristics at different temperatures. (a-d) $V_{\text {drag }}-I_{\text {drive }}$ curves obtained at four typical temperatures by using setup 1 (see Figure S2a). Deviation of good linearity starts at $70 \mathrm{~K}$, while for $1.5 \mathrm{~K}$ clear universal conductance fluctuations are observed.

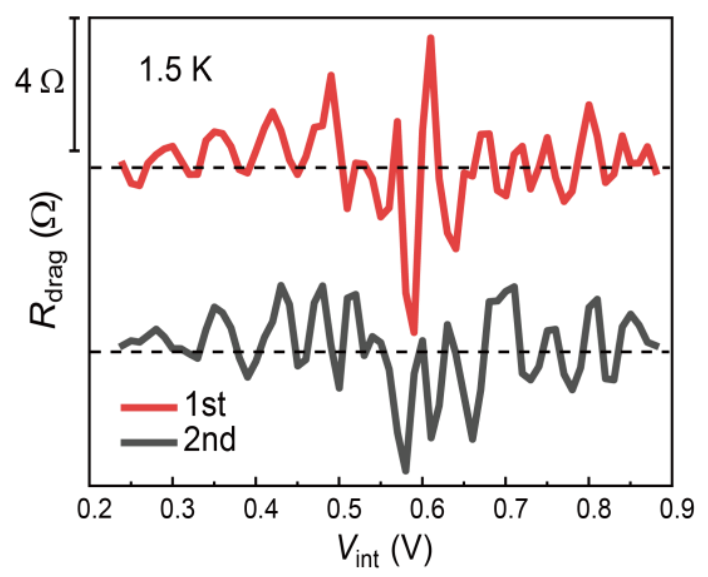

Figure. S4. $R_{\text {drag }}$ vs $V_{\text {int }}$ measured at $T=1.5 \mathrm{~K}$ for two different scans. $V_{\mathrm{BG}}$ is set as 0 $\mathrm{V}$ during the measurements. The black dashed lines correspond to the zero values of $R_{\text {drag. }}$ 


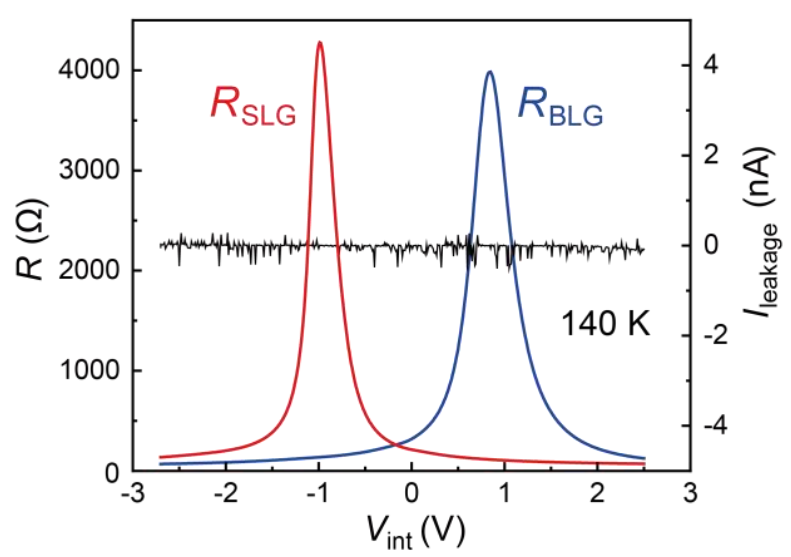

Figure S5. Field effect in SLG/BLG device while sweeping the interlayer-gate voltage $V_{\text {int. }}$ Layer resistance $\left(R_{\mathrm{SLG}}, R_{\mathrm{BLG}}\right)$ and interlayer leakage current ( $\left.I_{\text {leakage }}\right)$ vs $V_{\text {int }}$ measured at $140 \mathrm{~K}$.

(a)
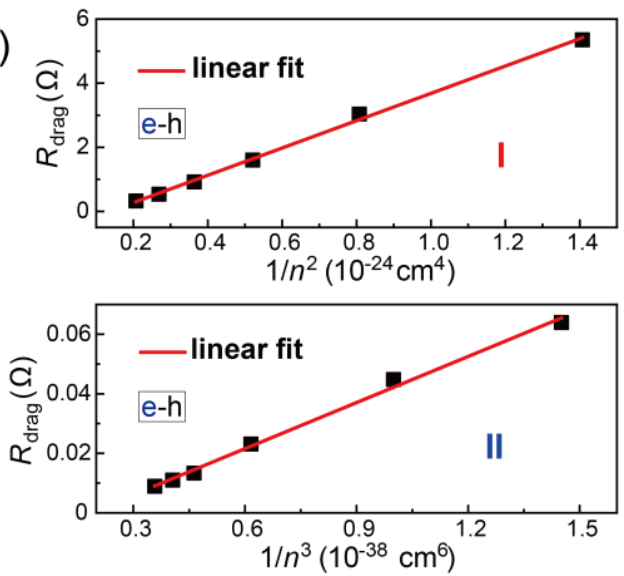

(b)

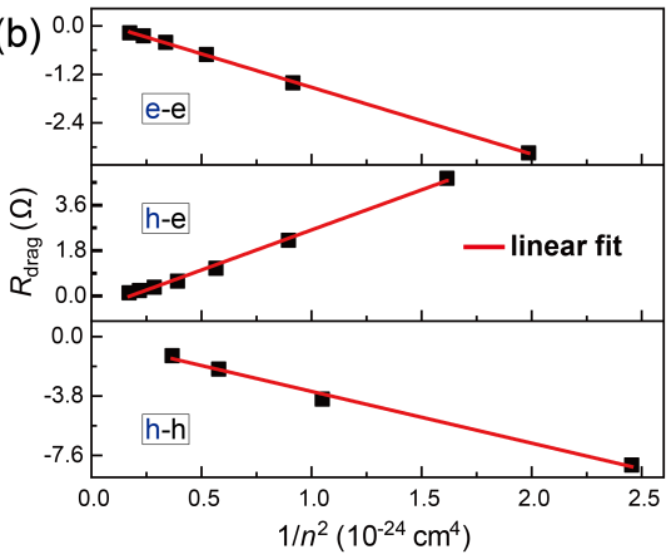

Figure S6. Carrier-density dependent characters of $R_{\text {drag }}$ along density matched lines. (a) Carrier-density dependence of $R_{\text {drag }}$ in e-h region (identical to the data showed in Figure $3 \mathrm{f}$ in the main text), which is linearly dependent on $1 / n^{2}$ in regime I and on $1 / n^{3}$ in regime II. (b) Carrier-density dependence of $R_{\text {drag }}$ along density matched line in the other three regions (e-e, h-e, h-h), identical $1 / n^{2}$ dependent character is observed within the low density regime.

TABLE S1. The extracted carrier density of BLG layer $\left(n_{\mathrm{B}}\right)$ and SLG layer $\left(n_{\mathrm{S}}\right)$ via Hall measurements for different $V_{\mathrm{BG}}$ at $V_{\text {int }}=0 \mathrm{~V}$ and $V_{\text {int }}=-0.8 \mathrm{~V}$. The Hall measurements were conducted at $140 \mathrm{~K}$.

\begin{tabular}{|c|c|c|c|c|c|c|}
\hline \multirow{3}{*}{$V_{\text {int }}(\mathrm{V})$} & & \multicolumn{2}{|c|}{ e-e region $\left(10^{12} \mathrm{~cm}^{-2}\right)$} & \multirow{2}{*}{ e-h region $\left(10^{12} \mathrm{~cm}^{-2}\right)$} \\
\cline { 3 - 4 } \cline { 5 - 6 } & $V_{\mathrm{BG}}(\mathrm{V})$ & $n_{\mathrm{B}}$ & $n_{\mathrm{S}}$ & $V_{\mathrm{BG}}(\mathrm{V})$ & $n_{\mathrm{B}}$ & $n_{\mathrm{S}}$ \\
\hline \multirow{3}{*}{0} & 60 & -5.476 & -3.045 & -110 & 4.469 & -1.970 \\
\cline { 2 - 7 } & 40 & -4.306 & -2.919 & -100 & 3.884 & -2.033 \\
\cline { 2 - 7 } & 20 & -3.136 & -2.792 & -80 & 2.714 & -2.160 \\
\cline { 2 - 4 } & 0 & -1.966 & -2.666 & -60 & 1.544 & -2.286 \\
\hline
\end{tabular}




\begin{tabular}{|c|c|c|c|c|c|c|}
\hline \multirow{3}{*}{-0.8} & \multirow{3}{*}{0} & \multicolumn{2}{|c|}{ e-e region $\left(10^{12} \mathrm{~cm}^{-2}\right)$} & \multirow{3}{*}{-110} & \multicolumn{2}{|c|}{$\mathrm{h}-\mathrm{h}$ region $\left(10^{12} \mathrm{~cm}^{-2}\right)$} \\
\hline & & $n_{\mathrm{B}}$ & $n_{\mathrm{S}}$ & & $n_{\mathrm{B}}$ & $n_{\mathrm{S}}$ \\
\hline & & -3.803 & -0.466 & & 2.633 & 0.230 \\
\hline
\end{tabular}

\section{Supplementary references:}

(1) Casiraghi, C.; Hartschuh, A.; Lidorikis, E.; Qian, H.; Harutyunyan, H.; Gokus, T.; Novoselov, K. S.; Ferrari, A. C. Rayleigh Imaging of Graphene and Graphene Layers. Nano Lett. 2007, 7, 2711-2717.

(2) Ferrari, A. C.; Meyer, J. C,; Scardaci, V.; Casiraghi, C.; Lazzeri, M.; Mauri, F.; Piscanec, S.; Jiang, D.; Novoselov, K. S.; Roth, S.; Geim, A. K. Raman Spectrum of Graphene and Graphene Layers. Phys. Rev. Lett. 2006, 97, 187401.

(3) Ferrari, A. C.; Basko, D. M. Raman spectroscopy as a versatile tool for studying the properties of graphene. Nat. Nanotechno. 2013, 8, 235.

(4) Wang, L.; Meric, I.; Huang, P. Y.; Gao, Q.; Gao, Y.; Tran, H.; Taniguchi, T.; Watanabe, K.; Campos, L. M.; Muller, D. A.; Guo, J.; Kim, P.; Hone, J.; Shepard, K. L.; Dean, C. R. One-Dimensional Electrical Contact to a Two-Dimensional Material. Science 2013, 342, 614-617.

(5) Narozhny, B. N.; Aleiner, I. L. Mesoscopic Fluctuations of the Coulomb Drag. Phys. Rev. Lett. 2000, 84, 5383.

(6) Price, A. S.; Savchenko, A. K.; Narozhny, B. N.; Allison, G.; Ritchie, D. A. Giant Fluctuations of Coulomb Drag in a Bilayer System. Science 2007, 316, 99-102.

(7) Kim, S.; Jo, I.; Nah, J.; Yao, Z.; Banerjee, S. K.; Tutuc, E. Coulomb drag of massless fermions in graphene. Phys. Rev. B 2011, 83, 161401.

(8) Kim, S.; Tutuc, E. Coulomb drag and magnetotransport in graphene double layers. Solid State Commun. 2012, 152, 1283-1288.

(9) Gamucci, A.; Spirito, D.; Carrega, M.; Karmakar, B.; Lombardo, A.; Bruna, M.; Pfeiffer, L. N.; West, K. W.; Ferrari, A. C.; Polini, M.; Pellegrini V. Anomalous lowtemperature Coulomb drag in graphene-GaAs heterostructures. Nat. Commun. 2014, 5 , 5824 .

(10) Gorbachev, R. V.; Geim, A. K.; Katsnelson, M. I.; Novoselov, K. S.; Tudorovskiy, T.; Grigorieva, I. V.; MacDonald, A. H.; Morozov, S. V.; Watanabe, K.; Taniguchi, T.; Ponomarenko, L. A. Strong Coulomb drag and broken symmetry in double-layer graphene. Nat. Phys. 2012, 8, 896. 
(11) Narozhny, B. N.; Aleiner, I. L.; Stern, A. Mesoscopic Fluctuations of the Coulomb Drag at v=1/2. Phys. Rev. Lett. 2001, 86, 3610.

(12) Kim, S.; Jo, I.; Dillen, D. C.; Ferrer, D. A.; Fallahazad, B.; Yao, Z.; Banerjee, S. K.; Tutuc, E. Direct Measurement of the Fermi Energy in Graphene Using a DoubleLayer Heterostructure. Phys. Rev. Lett. 2012, 108, 116404.

(13) Min, H.; Sahu, B.; Banerjee, S. K.; MacDonald, A. H. Ab initio theory of gate induced gaps in graphene bilayers. Phys. Rev. B 2007, 75, 155115.

(14) Oostinga, J. B.; Heersche, H. B.; Liu, X.; Morpurgo, A. F.; Vandersypen, L. M. K. Gate-induced insulating state in bilayer graphene devices. Nat. Mater. 2008, 7, 151.

(15) Zhang, Y.; Tang, T.-T.; Girit, C.; Hao, Z.; Martin, M. C.; Zettl, A.; Crommie, M. F.; Shen, Y. R.; Wang, F. Direct observation of a widely tunable bandgap in bilayer graphene. Nature 2009, 459, 820.

(16) Li, J. I. A.; Taniguchi, T.; Watanabe, K.; Hone, J.; Levchenko, A.; Dean, C. R. Negative Coulomb Drag in Double Bilayer Graphene. Phys. Rev. Lett. 2016, 117, 046802. 